\section{Predicting recurrence in patients with breast cancer}

Adjuvant systemic therapy is recommended for the prevention of recurrence in most patients with lymph-node-negative breast cancer, but some individuals might not require such aggressive treatment. Wang and colleagues have identified a 'genetic signature' which could help to prevent overtreatment by identifying patients at low risk of distant metastases.

Using microarray technology, Wang et al. analyzed the expression of 22,000 genes in tumor samples from 115 lymph-node-negative breast cancer patients who had not received systemic adjuvant or neoadjuvant therapy. A set of 76 genes was identified, the expression levels of which were correlated with survival free of distant recurrence. Most of these were well-characterized genes and several different functional classes were represented.

Testing the 76-gene profile in an additional 171 patients revealed a sensitivity of $93 \%$ and a specificity of $48 \%$ for the identification of patients who developed distant metastases within 5 years. The signature remained prognostic when premenopausal and postmenopausal women were analyzed separately, and was also independent of age, tumor size, tumor grade and estrogen-receptor status. Importantly, this also applied to patients with small (10-20 mm) tumors.

On the basis of this analysis, genetic profiling using the 76-gene signature would recommend against unnecessary systemic adjuvant therapy in $48 \%$ of low-risk patients. This compares favorably with current guidelines, which result in around $90 \%$ of these patients receiving such treatment. If confirmed in further studies, say Wang et al., the genetic signature might rule out needless treatment in a substantial proportion of patients.

Original article Wang Y et al. (2005) Gene-expression profiles to predict distant metastasis of lymph-node-negative primary breast cancer. Lancet 365: 671-679

\section{Advances in immunotherapy for chronic myeloid leukemia}

Early results from an observational trial in Italy suggest that a new vaccine might improve outcomes in patients with chronic myeloid leukemia.
The multipeptide vaccine, named CMLVAX100, was designed to target the b3a2 splice-region variant of the $B C R$ - $A B L$-derived fusion protein p210, expression of which is specific to chronic myeloid leukemia. Sixteen patients with persistent, stable, b3a2-related chronic myeloid leukemia each received six subcutaneous vaccinations every 2 weeks. All patients continued to receive conventional treatment with imatinib or interferon-alpha (IFN- $\alpha$ ).

The vaccine was well tolerated and appeared to generate an antitumor effect in the majority of patients. After 3 months, improved cytogenetic responses were observed in all nine imatinib-treated patients who had cytogenetic disease at baseline, and in five of the six patients receiving IFN- $\alpha$. A total of seven patients achieved complete cytogenetic remission. Furthermore, a molecular responseindicated by a failure to detect $B C R-A B L$ transcripts-was observed in four of these patients. The one imatinib-treated patient who began the study in complete cytogenetic remission also showed a reduction in residual molecular disease after receiving the vaccine.

The investigators conclude that vaccination with CMLVAX100 might help to reduce residual disease in patients with b3a2-related chronic myeloid leukemia, and might also improve molecular response rates. The results of larger studies are awaited.

Original article Bocchia M et al. (2005) Effect of a p210 multipeptide vaccine associated with imatinib or interferon in patients with chronic myeloid leukaemia and persistent residual disease: a multicentre observational trial. Lancet 365: $657-662$

\section{How do we care for patients with malignant glioma?}

Although glioma is the most common primary brain tumor, little is known about patterns of care in patients with these tumors. The Glioma Outcomes project was set up to address this problem by prospective recording of the clinical presentation, treatment and outcomes of a cohort of adults with primary grade 3 or 4 glioma. Results from the project have recently been published.

A total of 788 patients, from 52 clinical sites in the US, were recruited between December 